\title{
Reconfigurable Web Wrapper Agents for Biological Information Integration
}

\author{
Chun-Nan $\mathrm{Hsu}^{\dagger}$, Chia-Hui Chang ${ }^{\ddagger}$, Chang-Huain Hsieh ${ }^{b}$, Jiann-Jyh Lu ${ }^{\dagger}$, Chien-Chi Chang ${ }^{\natural}$ \\ ${ }^{\dagger}$ Institute of Information Science, Academia Sinica, Nankang, Taipei, Taiwan 105 \\ $\ddagger$ Dept. of Comp. Sci. and Info. Eng., National Central University, Chung-Li, Taiwan 320 \\ ${ }^{b}$ National Center for High-Performance Computing, Hsinchu, Taiwan, 300 \\ ${ }^{\natural}$ DeepSpot Intelligent Systems, Inc., Taipei, Taiwan, 104
}

\begin{abstract}
A variety of biological data is transferred and exchanged in overwhelming volumes on the World Wide Web. How to rapidly capture, utilize and integrate the information on the Internet to discover valuable biological knowledge is one of the most critical issues in bioinformatics. Many information integration systems have been proposed for integrating biological data. These systems usually rely on an intermediate software layer called wrappers to access connected information sources. Wrapper construction for Web data sources is often specially hand coded to accommodate the differences between each Web site. However, programming a Web wrapper requires substantial programming skill and is time-consuming and hard to maintain. This paper provides a solution for rapidly building software agents that can serve as Web wrappers for biological information integration. We define an XMLbased language called WNDL, which provides a representation of a Web browsing session. A WNDL script describes how to locate the data, extract the data and combine the data. By executing different WNDL scripts, user can automate virtually all types of Web browsing sessions. We also describe IEPAD, a data extractor based on pattern discovery techniques. IEPAD allows our software agents to automatically discover the extraction rules to extract the contents of a structurally formatted Web page. With a programming-by-example authoring tool, a user can generate a complete Web wrapper agent by browsing the target Web sites. We built a variety of biological applications to demonstrate the feasibility of our approach. The software is available at http://chunnan.iis.sinica.edu.tw/software.html or by contacting the authors.
\end{abstract}

Keywords: Wrappers, software agents, WNDL, IEPAD, information extraction, information integration, bioinformatics, World Wide Web.

\section{Introduction}

Advances in DNA sequencing and genome mapping techniques allow the Human Genome Project to be completed earlier than expected and result in rapidly growing databases of genomic and proteomic sequences. A variety of biological data, including DNA and amino acid molecular sequences, 3D-structure data of huge molecules, images, documents, and reports of experiments and analysis, in overwhelming volumes are made available for public access on the World Wide Web. The Molecular Biology Database Collection [4], an online data source that lists key databases of value to the biological community, currently holds over 335 biological data resources on the Web, up from 281 the year before (2001). The total number of all available databases is estimated to reach 600 . These databases include huge collections 


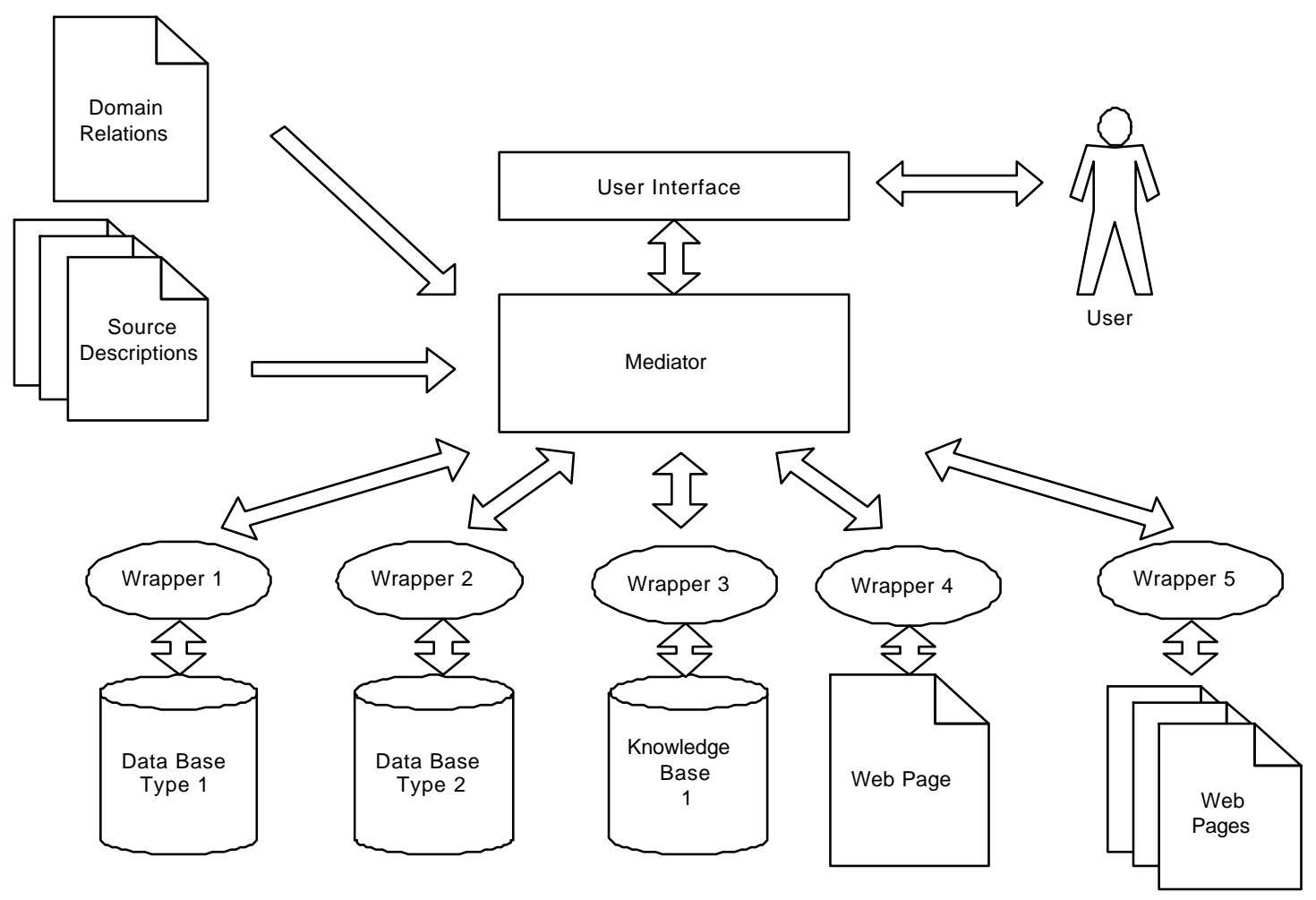

Figure 1: Architecture of Information Integration System

of the results of systematic sequencing as well as many that provide new value to the underlying data by virtue of curation by experts in a speciality or provide new types of data associations to facilitate knowledge discovery. Searching and integrating the information on the Web will become an indispensable biological experimental technique and is one of the most critical issues in bioinformatics.

Many solutions have been proposed to address this issue. One of the solutions is to develop information integration systems that are generally built with the architecture depicted in Figure 1 . The information integration system provides its users a single cohesive view with seamless integrated information that cannot be easily accomplished by a single information provider. Examples of biological information integration systems include SRS [5] and many other systems described in [23]. Other work include [13] and [12]. Genebank [14] databases hosted by the National Center of Biotechnology Information (NCBI) of the United States can be considered as the largest information integration system with more than ten databases interlinking with each other via hyperlinks.

Information integration systems allow their users to formulate their queries in domain relation terms defined in advance to describe a target application domain. Then a mediator [36] will construct a query plan to decompose the user query into subqueries to external data sources and determine execution and data flow orders of the subqueries. Mediators rely on wrappers to allow for transparent access to the data sources. Wrappers serve as the translators between the mediator and the data sources. Therefore, 
a wrapper is required for each type of data sources. Since the largest and the most up-to-data biological data sources are on the Web, building wrappers for Web data sources is important.

Web information integration is different from database information integration due to the nature of the Web, where data are contained in interlinked Web pages rather than tables or objects with clearly defined schema as in database systems. Building wrappers for relational and object databases is relatively easy because they are ready for access by another program. However, since the biological databases usually only open to the public through the Web, a wrapper for the Web is required to access those data sources. Web wrappers must automate Web browsing sessions to extract data from the contents of the target Web pages. But each Web site has its particular page linkages, layout templates, and syntax. A brute-force solution is to program a wrapper for each particular browsing session. That solution, however, may lead to wrappers that are sensitive to Web site changes and thus may become difficult to scale up and maintain. Our solution emphasizes the reconfigurability of the Web wrappers so that they can be rapidly developed and easily maintained without skillful programmers. This is particularly important for life science researchers not specialized in programming.

The remainder of this section discuss the issues involved in the development of reconfigurable Web wrappers for biological information integration.

\subsection{Web Site Navigation Problem}

Most of previous Web information integration systems such as Ariadne [21] simply model a Web page as a data source relation and ignore the necessity of navigation between Web pages. They did not capture the relationships between the linked data and Web pages under the assumption that all Web pages can be obtained with a single HTTP request. Another Web information integrator called ShopBot [11] did not address this problem explicitly.

In order to fetch a Web page, many kinds of interaction with the Web server might be required, for example, browsing through static URL links or querying by dynamic URLs. In addition to URLs, other information such as base URL, CGI parameters, cookie object, user authentication, etc, may be required. As these values may only be obtainable from a sequence of interactions between the user and the Web server, specifying these parameters with constant values in advance cannot solve the problem. Software agents are therefore needed to simulate the browsing processes and compose appropriate HTTP requests to locate the pages. Previous work in Web information integration either only offer hyperlinking or require custom-made program codes, which may impede applicability and maintainability of the system.

This paper provides a solution using an XML[35]-based language, called WNDL (Web Navigation Description Language), which will be presented in Section 3. Scripts written in WNDL are interpreted and executed by a WNDL executor, which offers the following features:

1. declaratively represent complex navigation and data gathering behavior of a user session,

2. XML format that eases information interchange between applications,

3. accumulate and integrate data extracted from Web pages along the traversal,

4. handle dynamically generated hyperlinks and CGI query HTML forms, 
5. tolerate mal-formed HTML documents.

\subsection{Data Extraction Problem}

To allow for postprocessing of the data obtained from the World Wide Web, it is necessary to extract and formulate the contents of Web pages into structured and machine-readable formats. Though XML is designed for this purpose, most of biological data sources still render the data in HTML for human browsing. Due to the daily increasing amount and format changing frequency of Web pages, it will be tedious, time-consuming, and error-prone to use custom-made programs to extract data from Web pages. Therefore, it is necessary to generate a Web page extractor automatically or semi-automatically. Several algorithms have been developed to address this problem by wrapper induction, including the work by Kushmerick [22], Muslea [25], and Hsu [18]. Wrapper induction systems apply machine learning techniques to induce Web data extractors with human labeled (annotated) training examples. The training examples demonstrate a wrapper induction system how to segment a Web page and group segmented strings into attributes and data records. Given the training examples, the wrapper induction system generates a specialized extractor for each Web data source. Their work produce accurate extraction results, but still require noticeable human intervention.

An early prototype of our system is equipped with a wrapper induction system called SoftMealy [17, 18] to generate data extractors. Recently, we have developed another algorithm called IEPAD (an acronym for information extraction based on pattern discovery) [6, 7]. Unlike the work discussed above, IEPAD applies sequential pattern mining techniques to discover data extraction patterns from a document. This removes the need of labeling training examples and thus minimizes human intervention. There are some heuristic-based work on the market that claim to be able to extract data from the Web automatically. However, these work are limited to a very narrow class of Web pages that matches their heuristics. In contrast, IEPAD does not depend on heuristics. IEPAD will be described in details in Section 4 .

A complete Web wrapper agent includes a WNDL script as well as IEPAD data extractors. The expressive power of WNDL and IEPAD is expected to cover the need of most Web information integration applications in biology. We also developed a programming-by-example authoring tool which allows users to generate a Web wrapper agents by browsing the target Web sites for their particular information gathering task. The generated Web wrapper agent can be reconfigured through the same authoring tool to maximize the maintainability and scalability for a biological information integration system.

\subsection{Organization of this Paper}

In Section 2, we describe some example applications that we built to demonstrate the utility and feasibility of WNDL Web wrapper agents in bioinformatics. Section 3 presents the definition of the language WNDL. Section 4 explains IEPAD. Related work are surveyed and compared in Section 5. Finally, Section 6 summarizes our work. 


\section{Applications of Web Wrapper Agents}

This section presents three example applications of Web wrapper agents. The first example shows how to apply Web wrapper agents to automate a complex Web browsing session. The second example shows how to use Web wrapper agents to rapidly build an integrated database on a special research topic. The integrated database combines in-house data and online databases with visualization and analysis software tools. The third example shows how to apply Web wrapper agents to reduce workload of repeated Web browsing routines at biological labs.

\subsection{Searching SNPs in ESTs}

Expressed sequence tags (ESTs) are short nucleotide sequences that are considered as a shortcut to the alternative spliced, expressed forms of the genes. Their identification involves the isolation of messenger RNA (mRNA), the intermediate product between genes and proteins. The spectrum of mRNAs within a cell reflects the spectrum of active genes that generate protein. From a given mRNA, one can deduce the DNA sequence (called cDNA) from which it was expressed. The resulting partial gene sequence comprises an EST. dbEST, one of many Genebank databases hosted by NCBI [14], currently contains twelve million entries of EST (as of July 26, 2002) and is one of the largest and fastest growing biological databases. Many ESTs identified in the private sector have been patented because some of them may provide invaluable hints for interpreting genome sequences.

Meanwhile, single nucleotide polymorphism (SNP) markers are single base pair positions in genomic DNA at which different sequence alternatives (alleles) exist in normal individuals in some population(s), wherein the least frequent allele has an abundance of one percent or greater. With the looser "variation" definition, SNPs occur approximately once every 100 to 300 bases. SNP alleles can be used as genetic markers. Because the SNP itself is the variant that causes or contributes to the risk of developing a particular genetic disorder, SNPs are expected to facilitate large-scale association genetics studies. Identifying ESTs that contain a given SNP may shed new light on possible treatments of many genetic disorders. Therefore, enormous efforts have been devoted to associate SNPs with ESTs.

This example application regards an agent that automates the search of a set of known ESTs that contain a given SNP through the databases at NCBI. More precisely, given the reference cluster ID (or RS number) of a SNP (e.g., "rs1614984"), the agent is supposed to return all ESTs in dbEST that contain this SNP. One can browse the Web to obtain the search results. The browsing session requires the following steps to accomplish this task:

1. First, from dbSNP homepage (http://www.ncbi.nlm.nih.gov/SNP), enter the RS number to search the SNP data;

2. from the output Web page, extract the gene names and hyperlinks in "LocusLink" section;

3. follow each hyperlink, get the "UniGene" name and hyperlink in "Additional Links" section; 


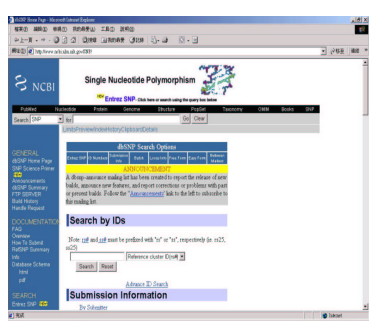

1.Enter SNP number

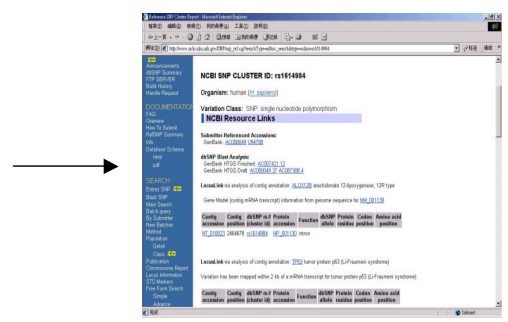

2. Get gene name and hyperlink

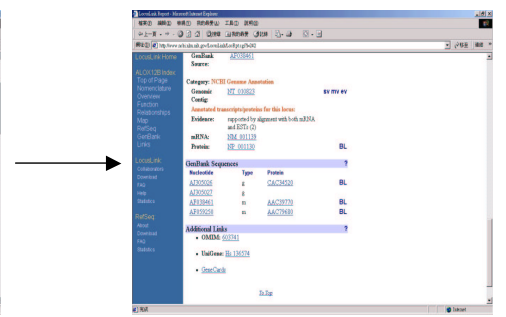

3. Follow each hyperlink, get UniGene name and hyperlink

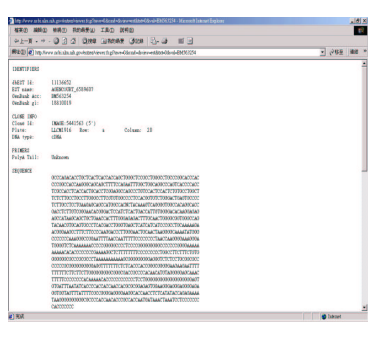

6. Detailed dbEST data

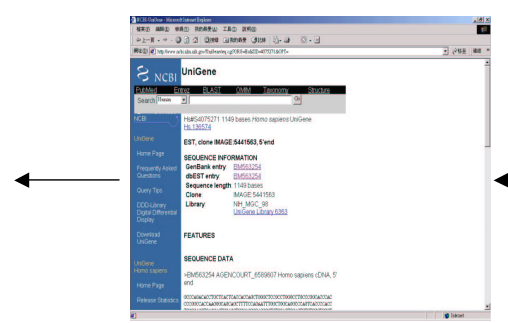

5. Get dbEST entry link

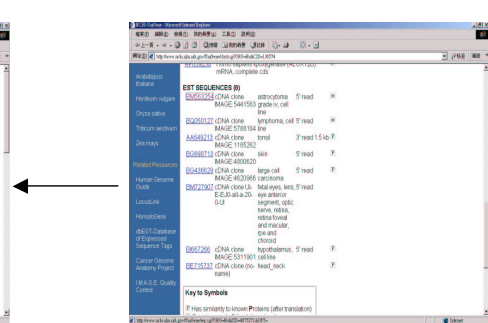

4. Get dbEST GeneBank acc number

Figure 2: Identifying ESTs that contain a given SNP by browsing the Web

4. follow the hyperlink, get the Genebank access number and hyperlink of ESTs in "EST SEQUENCES" section;

5. get the hyperlink to dbEST entry in "SEQUENCE INFORMATION" part;

6. lastly, extract the sequence in the dbEST entry page.

The above steps are illustrated in Figure 2. Note that except for the first step which starts from a static URL, all other steps involve dynamic URLs that must be obtained from each search result of previous step. In other words, each search result requires a data extractor to extract specified data for the use in the next step. In addition, step 3 to 6 must be repeated for every gene name obtained in Step 2. Therefore, it might take hundreds of interactions for a user to collect all known ESTs that contain a given SNP. For SNP "rs1614984", the output includes 289 EST entries, which may take several working days to complete by browsing the Web by hand, a laborious and tedious task.

To automate the process, one can generate a WNDL agent for this task through our authoring tool by demonstrating how to obtain one of the EST entries given a SNP. One browsing path is sufficient for our system to generate a WNDL script that generalizes to collect all intended EST entries as well as necessary data extractors. The output will be formulated into structured XML data records ready for 

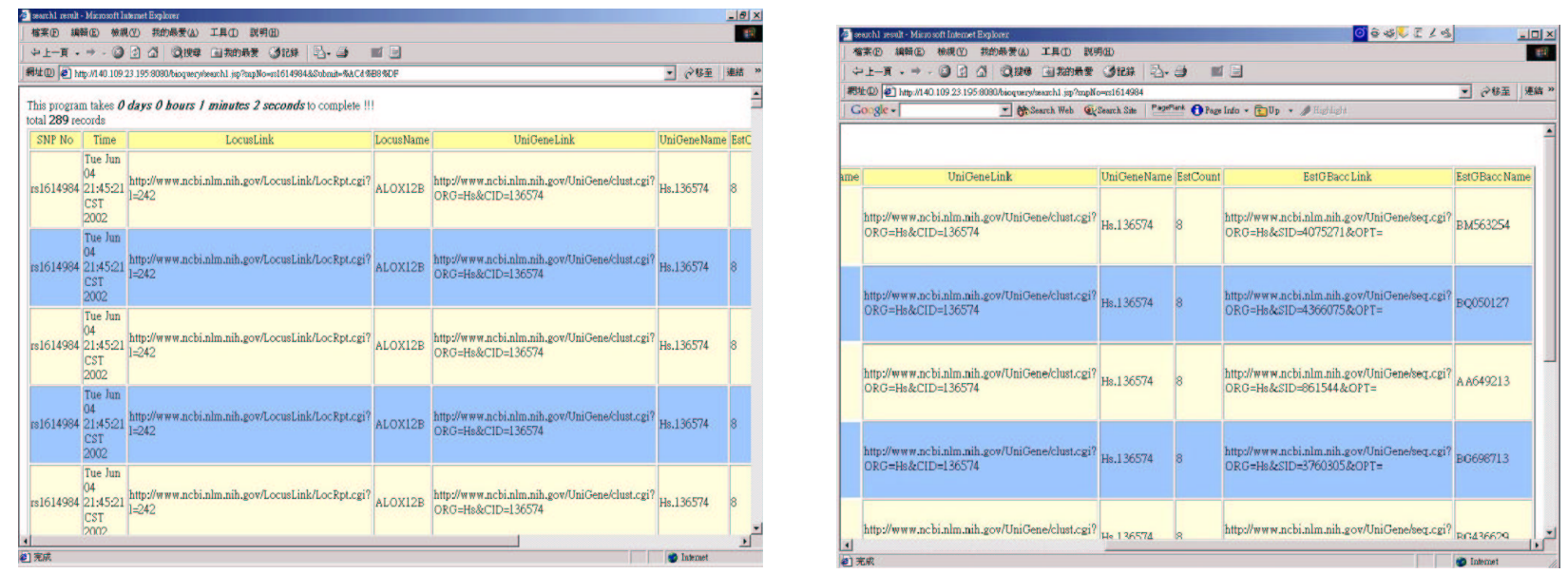

Figure 3: The output ESTs

postprocessing, as shown in Figure 3, where the output is formulated as an HTML encoded Web page. In this example, the output table has a total of nine attributes. The left window in Figure 3 shows the first six attributes and the right window shows the last five attributes. The agent can also take a list of SNPs as the input.

A common approach to such a task is to mirror dbEST and dbSNP (only flat files available) via FTP in advance and perform the search locally. That approach requires substantial programming skills and computing resources: large disk space (about 100 Giga bytes to store 12 million EST entries), a parser to parse flat files, a data normalizer to decompose parsed data into data tables that conforms the third normal form [34], and finally algorithms to identify ESTs that contain a SNP. In contrast, applying agent technologies requires much less programming skills and computing resources. Also, we can leverage available data connection established by NCBI between databases. Moreover, each execution of the agent collects the most recent results in dbEST submitted from all over the world. This is critical for biological databases that grow at a breakneck pace. For example, our agent now yielded 289 ESTs for SNP "rs1614984", up from 261 a week before ${ }^{1}$. With a timer and a redundant data filter, the agent can be extended to alert its users interesting updates in dbEST with new data entries collected as well.

\subsection{Integrated GAGs Database}

The second application is a small integrated database which contains annotated data of glycosaminoglycans (GAGs) and their binding proteins to facilitate research on the molecular mechanisms of glycoside in human living cells. A Web-based interface was constructed to allow biologists to access this integrated database through a Web browser. Figure 4 shows a snapshot of this system.

GAGs are linear polysaccharides composed of long repeating disaccharide units, which contain either of two modified sugars - N-acetylgalactosamine (GalNAc) or N-acetylglucosamine (GlcNAc), or one

\footnotetext{
${ }^{1}$ The major possible reason for getting so many entries is the redundancy in dbEST.
} 
of their derivatives. The major function of GAGs is the formation of a matrix to hold together the protein components of the skin and connective tissue of vertebrate animals. Through the presence of either sulfate or carboxylate groups, GAGs are mostly negatively charged and acidic. The specific GAGs of physiological significance are chondroitin sulfates in the cartilage, keratan sulfates in the cornea, dermatan sulfates of the skin and blood vessels, hyaluronic acid of the connective tissue, heparan sulfates in the lung, and heparin in the liver. For the basic chemical characteristics, classifications and structures of these GAGs, glucuronic acids and other related molecules, this integrated database simply stores the data locally, or hyperlinks to PDB [2] and SCOP [27] for more information.

The majority of GAGs in the body are linked to core proteins, forming proteoglycans, one of the protein post-translation modification types. Proteoglycans and GAGs perform numerous vital functions within the body. For related data about these binding proteins, we deploy a team of eleven Web wrapper agents to collect the data from online biological databases. These agents are triggered periodically to update our database with the most recent related textual and structural data. The online databases connected include NCBI PubMed [29], PDB, PIR [31], KEGG [26], SWISS-PROT and TrEMBL [33], Genebank [14], etc.

The collected 2D or 3D protein structure data (such as from PDB) can be fed into molecular viewers: RasMol [30] and Chime [8]. For instance, we can display the electric potential distribution and posttranslation modification sites of the protein to visualize the best fit for ligand-receptor binding site. The protein sequences can also be fed into protein BLAST [3] on the Web by an agent to perform similarity search. Both services can be accessed through the Web interface of the integrated database. With Web wrapper agents, an integrated database on another biological research topic can be constructed with abundant data from online databases in a similar manner.

\subsection{Microarray Analysis}

Our wrapper approach is not limited to handle academic biological data sources. As long as the data is available on the Web, we can rapidly build an agent and perform a variety of information integration task.

The last example application is for gene chips analysis (i.e., microarray) that involves a commercial Web service. Microarray analysis is to study the relative abundance of thousands of mRNAs obtained from complex biological data sets. In this application, our clients subscribe a Web-based microarray analysis service from Incyte [20], one of the largest companies specialized in microarray analysis. To use the service, they need to enter a designated ID and password and then submit their microarray data. However, the service usually returns a large number of gene sequences (about 10,000). As the number of microarray data to be analyzed increases, the comparison task becomes overwhelming. There is a need to automate the process and store the results in structured databases for further analysis. To solve their problem, they tried to write a program from the scratch. However, since the task requires experienced programmers for up to one thousand lines of $\mathrm{C}$ codes, including two layers of HTTP connections, 


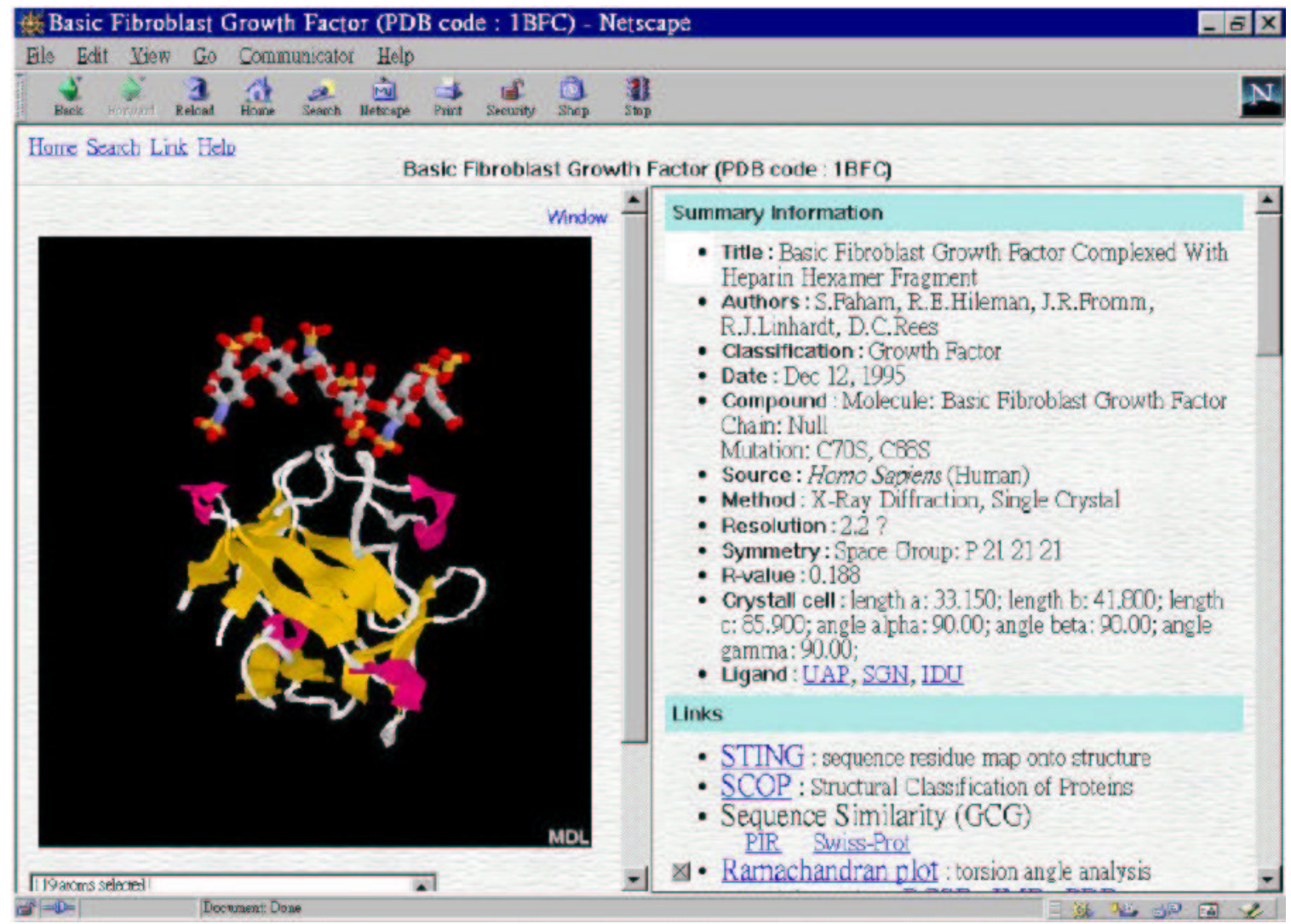

Figure 4: Snapshot of the integrated database of glycosaminoglycans and their binding proteins 
user authentication, data extraction, and ODBC database connection, they never really complete the program. With our tool, it requires only 48 lines of WNDL script and three data extractors to build up their own database. The whole process takes less than one working hour. With the wrapper agent they can focus on data mining from the database they collected.

In addition to biological databases, we are also applying the agents to build an integrated decision support system for the biotech industry by integrating online clinical trial databases, biotech news, patents, financial and marketing reports of biotech firms, etc.

\section{$3 \quad$ Web Navigation Description Language (WNDL)}

The Web Navigation Description Language (WNDL) is an application of eXtensible Markup Language (XML)[35] for describing a Web browsing session. This section presents the definition of WNDL, the executor that executes a script in WNDL, and the authoring tool that generates WNDL scripts.

Although the terminologies used in this section are primarily based on a working draft, Web Characterization Terminology $\&$ Definitions Sheet [9], from the World Wide Web Consortium (W3C), we reuse some of these terms and endow them with slightly different meanings. Their specific meanings in this context are defined as follows.

Definition 3.1 (Logical Web Site) A cluster of Web pages that are related to each other, each page contains certain amount of data. The data distributed among these pages can be integrated together and have a logical meaning.

Definition 3.2 (Web Page Class) A set of Web pages that a given data extractor can be applied to parse and extract their contents.

Though the definition depends on the expressive power of the given data extractor, a Web page class usually refers to a set of related Web pages generated by one single CGI program or Web pages with an identical layout template. For example, the output pages of PubMed's keyword search service comprise a Web page class.

\subsection{WNDL Definitions}

As all applications of XML, a WNDL script consists of a set of elements. Each element may have a set of attributes and subelements. In WNDL, a user session can be described by a data Web map (DWM), which is conceptually a directed graph with nodes and edges. The DWM map is the primary data container in a WNDL script. The information stored in DWM describes how to reach destined Web pages and how to extract the contents from those pages. The definitions of the subelements of DWM are enclosed in element Map. Subelements of Map include Entrance and one or more Node elements, and element Entrance contains a subelement Edge. The edge in element Entrance represents the way to access a logical Web site outside the scope of the defined DWM without further interaction with the 


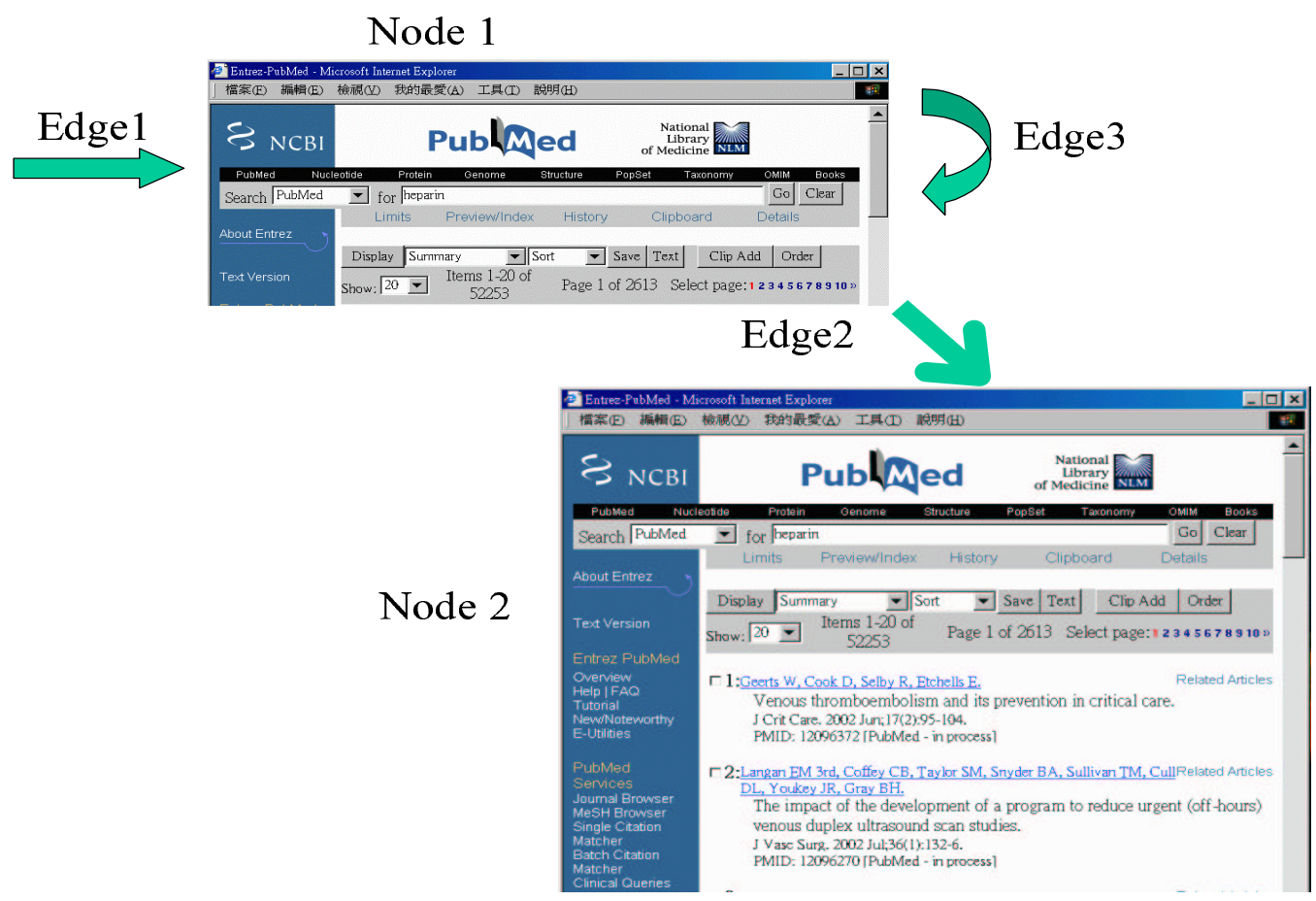

Figure 5: Conceptual illustration of the data Web map for PubMed

Web server. Typically, this leads to the front page of a Web data source. For example, the entrance to retrieve papers in PubMed is via the URL http://www.ncbi.nlm.nih.gov/PubMed, its front page.

In the following subsections, we will go through a complete example for modeling the browsing session of retrieving papers in the well-known online biomedical paper collection PubMed. The browsing session for PubMed can be perceived conceptually as a graph with two nodes and three edges as Figure 5 depicts.

\subsubsection{Data Web Map Edge}

An Edge in a DWM represents a possible mean to obtain a page that belongs to a Web page class denoted by the destination node of this edge. A DWM edge serves as the container of the necessary information of actual HTTP requests for both statically and dynamically generated HTML documents. The information for the requests consists of a set of parameters. Values of these parameters can be either specified in the WNDL script or bound during the run-time.

There are three edges in the example model. Edge 1 simulates the action of submitting a new query. Edge 2 simulates the action of browsing search results page by page numbered from 1 to 10 (each page contains 20 search results). Edge 3 simulates the action of jumping to the eleventh page. For most Web sites, usually a next page button leads to the following search results (say 21 to 40). However, PubMed provides a next button " $\rangle$ " which leads to search results 201 to 220 . To get the next twenty search results, we need to following the ten image links (numbered 1 to 10) one by one and then follow the 




Figure 6: Edges in the WNDL script for PubMed

button ") \" for the next 200 results if they exist. Note that unlike URL hyperlinks that can be usually seen in a Web page, the image links for next pages are IMAGE INPUT $\{$ "page 1" to "page 10" $\}$ of the form named "frmQueryBox."

The edges involved in the above browsing steps are encoded in WNDL as shown in Figure 6. Edge 1 is the entrance edge of this map that sends the query to get the resulting Web page, i.e., Node1 in this case. The URL attribute can be a constant or a variable. In WNDL, HTML forms are treated as parameterized URLs. Their parameters are specified in element QueryParam. Again, the value of QueryParam can be a constant or a variable. Note that some Web sites use a user session ID mechanism to recognize HTTP requests from an identical user to keep track of a user session. This mechanism helps Web servers to determine the contents of Web pages they should respond for each user. If such a mechanism is used, we have to start from a static link to extract the dynamically generated session ID instead of sending an HTTP request directly to obtain a destined page.

Once the first connection is successful, it leads us to the destination Node1. From Node1, we can continue the next connection to Node2 via Edge 2. As described above, Edge 2 simulates the action of browsing search results page by page. The HTTP connection information is embedded in the Web pages and can be extracted to bind the parameter values of Edge 2. In this case, since the values underlying the images of the page numbers are not URL links but image submission INPUT, the form that specifies the action CGI must be specified. The image submissions and the query form can be extracted and denoted by two variables, \&form1 and \&imglink, respectively. The connection can be specified by elements QueryForm and QueryParam. How the values of these variables are extracted for 
Node1 will be described in Section 3.1.2.

Edge 3 is an edge that has identical source and destination node as depicted in Figure 5. Therefore, it is a self-looping edge. Like Edge 2, the query type of Edge 3 is an HTML form, where QueryForm is specified by variable \&form2 and QueryParam refers to variable \&nextTen. During the run-time, Node1 will form a self-loop. As described above, virtually any logical browsing session can be expressed in WNDL.

Element Timeout is also a subelement of Edge. Timeout contains the control information of the event handling for timeouts. In WNDL, the number of retry attempts and the time interval between each attempt can be specified. The specified time interval is equal to the time bound of a timeout event. If all attempts fail, the executor of WNDL will throw an exception signal to its invocator.

\subsubsection{Data Web Map Node}

A DWM node represents one Web page class in a target logical Web site. Defined again here, a Web page class is a set of Web pages with similar layout templates such that one data extractor can be applied to successfully. A Web page class usually represents the pages that are generated by a CGI program. The number of Web pages that a CGI program can generate is innumerable.

In WNDL, each node is a container of data in the pages of a Web page class. The contents extracted from the Web page class of a node will be encoded as a database table, whose attributes must be defined in a schema in advance. For example, for the Web page class of Node2 shown in Figure 5, we want to extract the information of the retrieved papers into a table with the following four attributes: authors, title, source (where the paper published), and PMID (PubMed ID). Figure 7 shows how they are defined in WNDL (see the definition for Node2). Since each Web page contains twenty search results, the correct output for this node should be a table of twenty records with these four attributes.

For each attribute in a table, we can specify our option for HTML tags filtering (KeepAll, KeepLink, and NoTag). This determines the behavior of a built-in HTML tag filter in the executor of WNDL. WNDL also allows us to describe how to join two tables extracted from adjacent nodes for the output. That way, data extracted during the browsing session can be aggregated in user defined manners.

The data extractor for a DWM node is specified as the value of element ExtractRule. The data extractor must be declarative in the sense that its extraction rules must be allowed to replace for different Web page classes without changing the program codes. In our implementation, we apply SoftMealy $[17,18]$ and IEPAD (see Section 4) as the data extractors. Other declarative data extractors can be applied, too. The value of ExtractRule can be the raw text of a set of extraction rules or an external file, specified as the value of attribute File of this element.

In our PubMed example, there are two nodes in the map as shown in Figure 5. Node1 represents the result of the entrance connection and will be used to extract the paths to the next pages. Node2 represents query result pages returned from the search form of PubMed.

In Node1, the information we are interested is the $<$ Form $>$ HTML tag block in this page. Some Web 


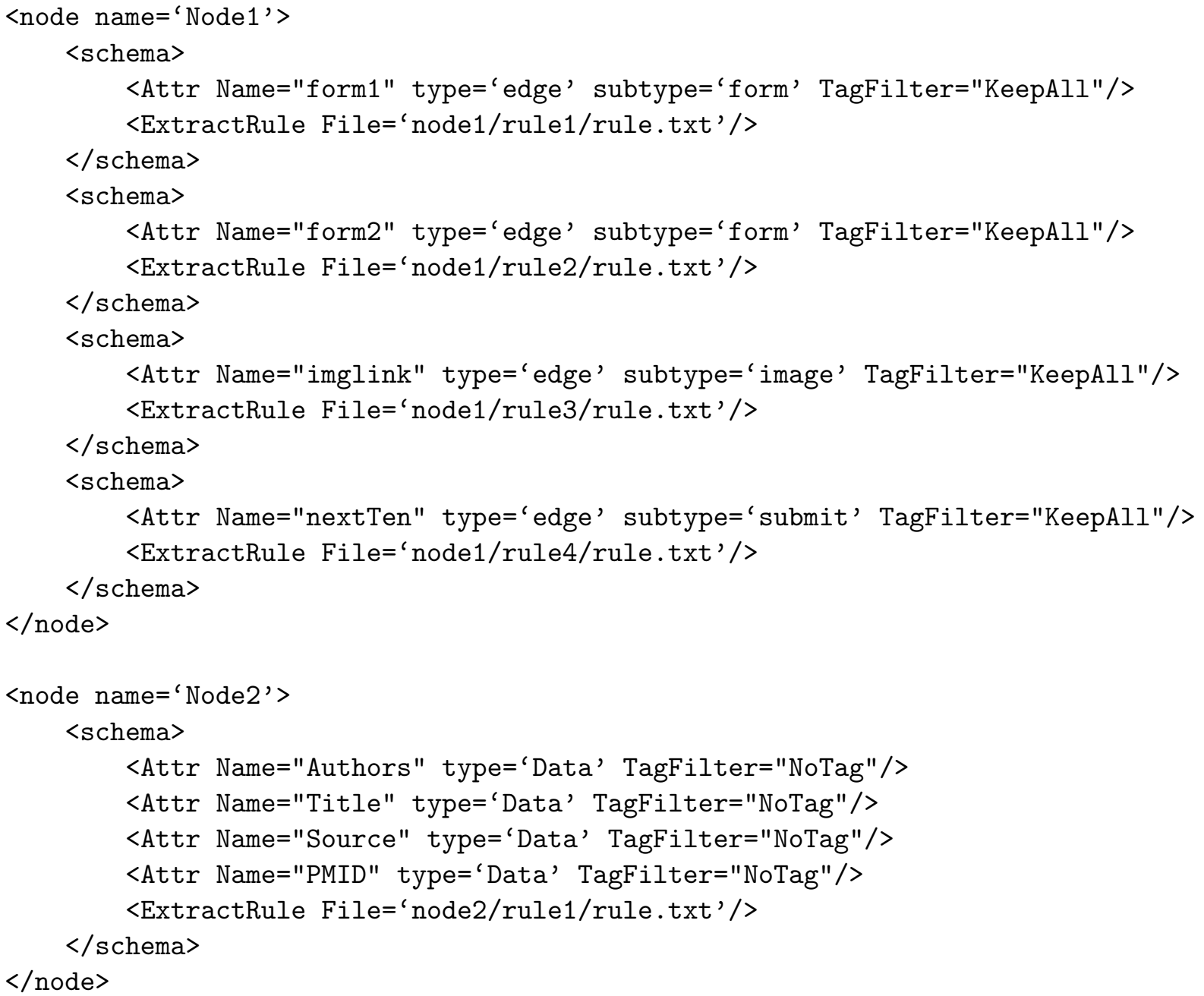

Figure 7: Nodes in the WNDL script for PubMed

sites use user session ID mechanism to recognize HTTP requests from identical user to keep track of a user session. This helps Web servers to determine the contents of the Web pages they should respond for different users accordingly. In some Web sites, HTTP clients (i.e., a browser) need this ID in order to continue navigation, whereas some Web sites use this ID optionally. Since PubMed does not belong to any of the above categories, the HTML query form can be extracted and used directly. If session ID is used, we have to start from a static link to extract the query form and the dynamically generated session ID for the following steps.

There are four sets of extraction rules for Node1. The first and second sets of the extraction rules extract the query form that contains the CGI program to the next page of the query results. Element Schema specifies that the extracted data will be bound to variables \&form 1 and \&form2. The third and the fourth sets of the extraction rules extract the INPUTs as the query parameters, which are bound to variables \&imglink and \&nextTen. Node2 represents the query results returned from the search engine of PubMed. The information we are interested in is the attributes of retrieved papers, including authors, 


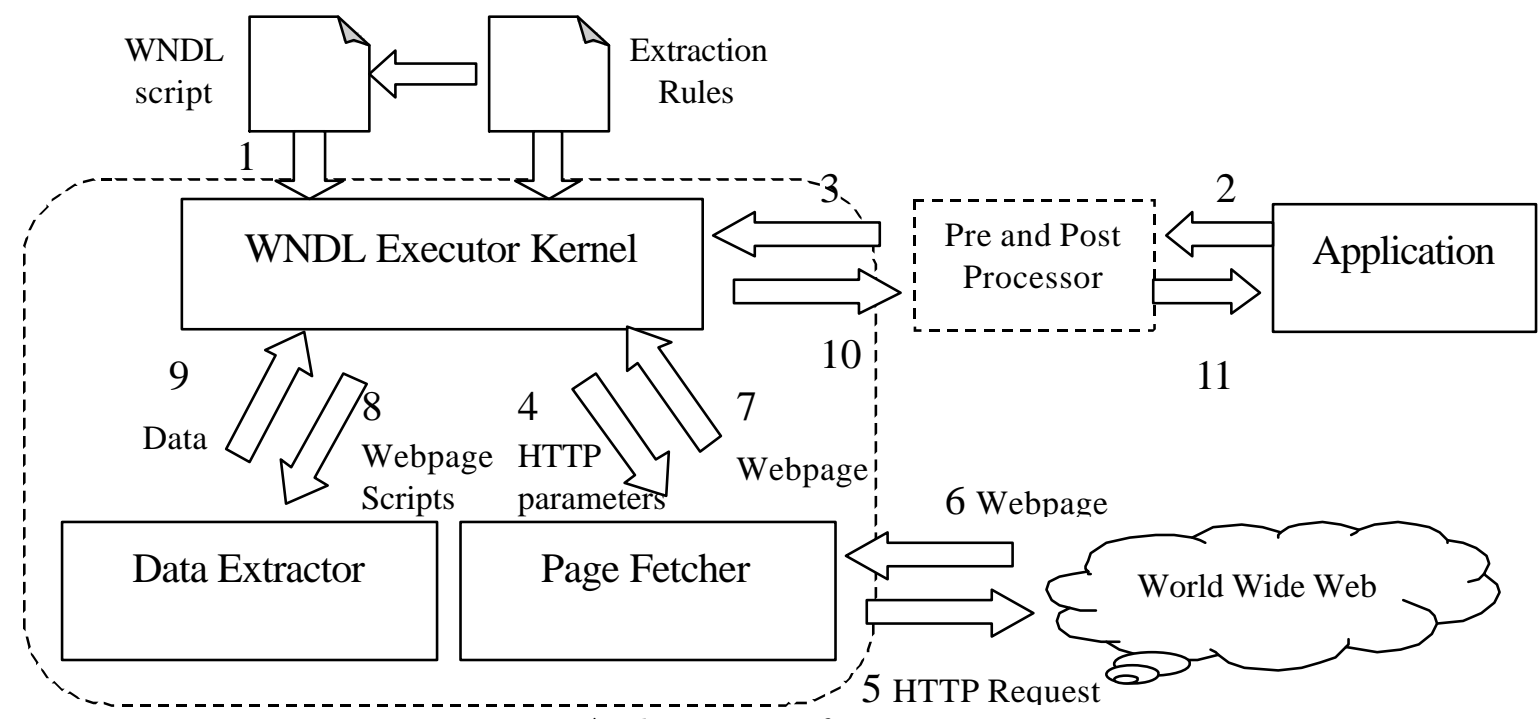

Figure 8: Architecture of WNDL Wrapper Executor

title, source, and PubMed ID. The complete WNDL script for PubMed is shown in Appendix.

The schema of the output data is specified in the extraction rules. The schema dictates which attributes defined in element Schema of the nodes will eventually appear in the output data table. In this example, the output data consists of a table with the four attributes defined in Node2. Section 3.3 explains how to specify the schema.

Composing extraction rules is not trivial and is another research problem itself. In Section 1, we have reviewed several systems designed to generate extraction rules from training examples. Section 4 presents our new approach from the perspective of pattern mining, which minimizes the need of human intervention in the generation of extraction rules.

\subsection{Architecture and Implementation of WNDL Executor}

The WNDL executor is composed of three components: executor kernel, page fetcher, and data extractor. Figure 8 shows the relationship between them and the order of execution steps. A WNDL script can be considered as the configuration file of a Web wrapper agent that wraps a logical Web site. The configuration file defines the behavior of the Web wrapper agent. During the execution, the executor kernel invokes the page fetcher and the data extractor according to the order specified in the DWM map, handles static information and variable binding information to complete a Web browsing session. The executor kernel maintains a pointer of the current state to traverse the DWM map. Basically, when the pointer points to an edge, the kernel invokes the page fetcher to obtain the next page and moves the pointer to the next node; when the pointer points to a node, the kernel invokes the data extractor and moves the pointer to the next edge.

The page fetcher abstracts HTTP connections to higher level interfaces for the executor. HTML 
and HTTP features that the page fetcher can handle include form element parsing, GET and POST HTTP methods, cookies, timeout, user authentication and mal-formed URL handling. The page fetcher transforms the parameters received from the executor into low level, executable HTTP requests. After actually obtaining a Web page from a Web server, the page fetcher sends this page back to the executor kernel directly. The executor kernel then feeds this page and the corresponding extraction rules to the data extractor. One page may go through this process multiple times if there are more than one set of the extraction rules required for this page. The extracted data will be returned to the executor for further processing.

\subsection{WNDL Authoring Tool}

In the early version of WNDL [19], the script is designed to be written by programmers. In this version of WNDL, the script can be generated automatically by an authoring tool. This authoring tool allows a user to generate a WNDL script in a programming-by-example manner, that is, the user simply browse the Web to show the authoring tool an example user session and the authoring tool will generalize the example into a WNDL script that describes this user session. Figure 9 shows a snapshot of its interface after generating the complete WNDL script for PubMed. The authoring tool is equipped with IEPAD (See Section 4) and the wrapper induction system Softmealy $[18,17]$ to generate extraction rules for the data extractors. With this authoring tool, it takes only four steps to generate a WNDL script.

1. Open the front page of the target Web site by specifying its URL as using a Web browser;

2. Create nodes by clicking the "Add Nodes" button when browse to a new Web page class;

3. Invoke IEPAD to generate extraction rules for each node;

4. If more than one node is needed, go back to step 2 .

As shown on the left frame of Figure 9, the example script contains two nodes with five sets of extraction rules.

We can create the edges as described below. The first edge is created to reach Node1. This is accomplished by clicking the submit button with parameters term and db set to value "aids" and "PubMed". The submission of this query is monitored by the system and compared to all forms contained in the front page of NCBI. The submitted parameters are recorded in QueryParam, which can be a constant value specified in the script or a variable bound to other query terms specified by the user during the execution time. For Node1, we also need to generate four extraction rules as well as specifying its schema. Each attribute in the schema is either of type Edge or Data. A Data attribute will appear in the final output, while an Edge attribute can be one of the four types: static link, form, submit button or image button. An edge can be created by a static link or a form with submit/image INPUT. For each Edge attribute, the user has to specify the destination node. Two Edge attributes pointing to the same destination can be combined to create a dynamic edge. Node2 is created similarly. 


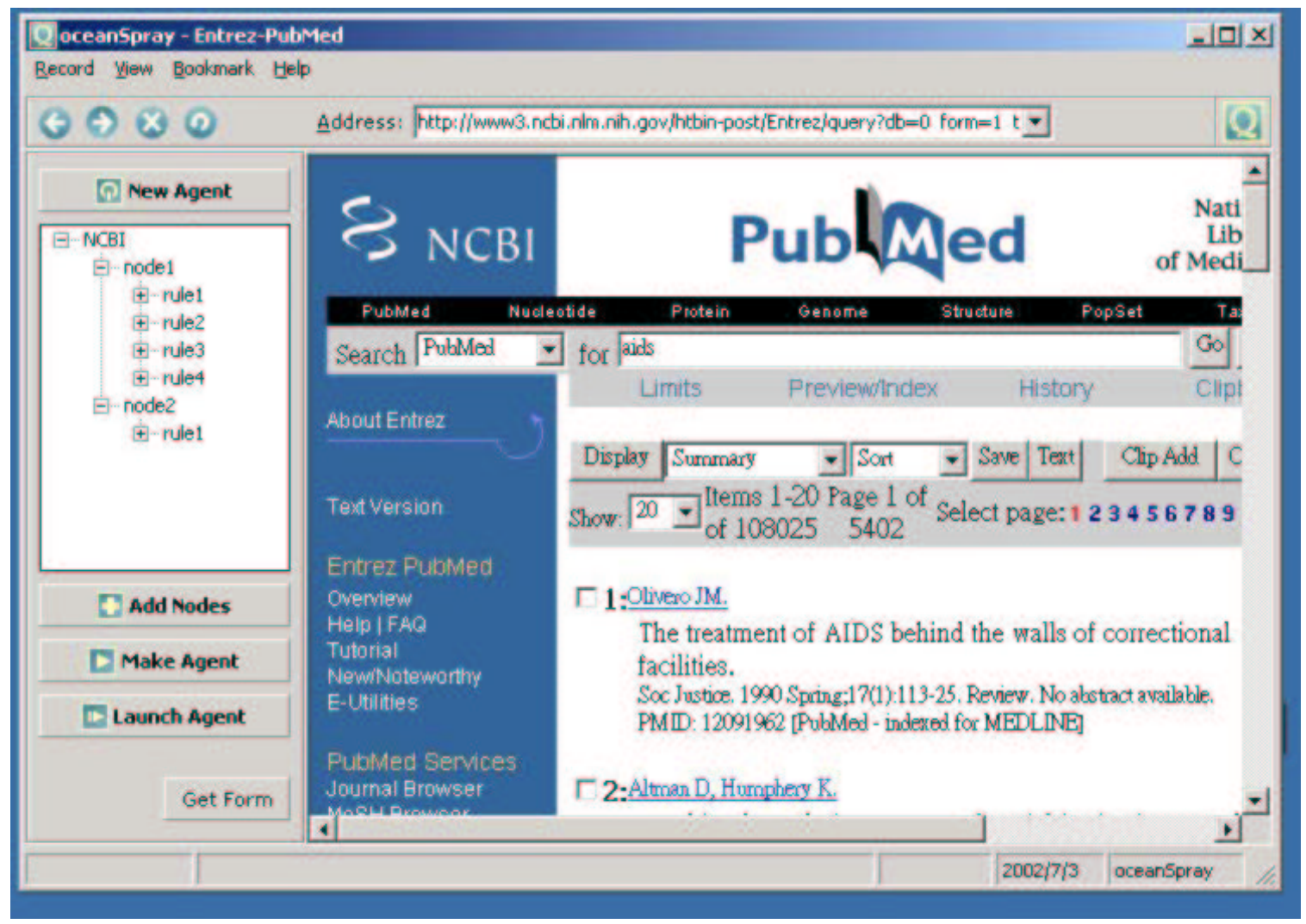

Figure 9: Snapshot of the authoring tool of WNDL

Once all the nodes and edges are specified, the complete WNDL script can be generated by clicking the "Make Agent" button. The authoring tool also provides a "Launch Agent" button for invoking the executor to test the generated WNDL script.

\section{Information Extraction based on Pattern Discovery (IEPAD)}

IEPAD is an acronym for "Information Extraction based on PAttern Discovery" which features the generalization of extraction patterns without user-labeled examples. This feature is based on the assumption that the input is a multiple-record Web page so that sequential pattern mining can be applied to discover the repeats. The system includes a pattern discoverer, which applies sequence mining techniques to discover possible patterns, and a multi-level analyzer, which conducts several multiple string alignments for attribute extraction. IEPAD discovers extraction rules for the WNDL executor to extract data from input Web pages. A user interface has been implemented and integrated with the authoring tool of WNDL for users to generate extraction rules of Web pages. Figure 10 shows a snapshot of the user interface of IEPAD. 


\subsection{Pattern Discoverer}

\subsubsection{Document Encoding}

The first step to reveal patterns in a Web page is to apply an encoding scheme to translate the Web page into a token string of abstract representation. There are various ways to encode a Web document. Different encoding granularities may reveal different patterns in a document. We can choose an encoding scheme based on the granularity level of the desired information to be extracted. For example, skipping all HTML tags will translate the following HTML block

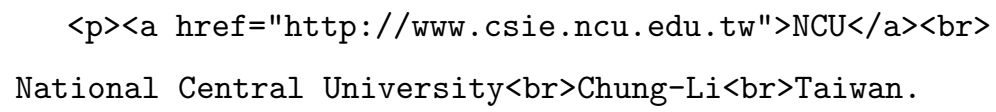

into

$$
"<\mathrm{P}><\mathrm{A}><\mathrm{TEXT}></ \mathrm{A}><\mathrm{BR}><\mathrm{TEXT}><\mathrm{BR}><\mathrm{TEXT}><\mathrm{BR}><\mathrm{TEXT}>\text { " }
$$

This process reveals the patterns of data records in a Web page. If there are $k$ entries in the Web page, then the above pattern will also occur $k$ times in the encoded token string. The idea behind our algorithm is to discover such patterns from the encoded token strings of a given Web page automatically. The discovered pattern can then be used as extraction rules.

\subsubsection{Constructing PAT Trees for Maximal Repeats}

Repeats are any substring that occurs at least twice in a string. To reduce the number of candidate patterns, the concept of maximal repeats is used to refer to the longest patterns. The idea is to extend a repeat in both directions to the most. We call a repeat left maximal (right maximal) if the repeat can not be extended on the left (right) direction (See [7]) for all occurrences. We say a repeat is maximal if it is both left maximal and right maximal.

To automatically discover repeats, a data structure called PAT trees is used to index all suffixes in the encoded token strings. A PAT tree is a Patricia tree (Practical Algorithm to Retrieve Information Coded in Alphanumeric [24]) constructed over all the possible suffix strings. A Patricia tree is a particular implementation of a compressed binary $(0,1)$ digital tree such that each internal node in the tree shows the different bit between suffix strings in the same subtree. Like a suffix tree [15], the Patricia tree stores all its suffix strings at the external nodes. For a token string with $n$ indexing point (or $n$ suffixes), there will be $n$ external nodes in the PAT tree and $n-1$ internal nodes. This makes the tree $O(n)$ in size.

PAT trees organize an input string such that all suffixes with the same prefix are stored in the same subtree. Hence, it provides surprisingly efficient, linear-time solutions to the problems of complex string search, including string prefix search, proximity search, range search, longest repetition search, most frequent search, etc $[15,24]$. Since every internal node in a PAT tree indicates a branch, the concatenation of the edge-labels on the path from the root to an internal node represents one right maximal repeat in the input string. 
Consequently, given the expected record count in a Web page and the minimum pattern length, we can simply traverse the PAT tree in postorder to enumerate all path-labels (from the root to an internal node) to discover all right maximal repeats. However, not every path-label represents a maximal repeat. At each internal node, we need to verify the left maximality by checking the left tokens of all leaves (suffixes). If all left tokens are the same, then this repeat is not left maximal and can be extended by another repeat, and we can simply discard it.

\subsubsection{Sifting For Regular and Contiguous Patterns}

As described above, dynamic pages are generated based on some predefined templates and relevant information is usually aligned regularly and contiguously. To discover the patterns of the layout templates, two measures, called variance and density, are defined to evaluate whether a maximal repeat is a promising pattern. In the following definitions, let $p_{i}$ be the position of the $i$-th occurrence of a maximal repeat $\alpha$ in an encoded token string such that $p_{1}<p_{2}<p_{3} \ldots<p_{k}$.

Definition 4.1 (Variance) Variance of a repeat is the ratio of the standard deviation of the interval between two adjacent occurrences $\left(p_{i+1}-p_{i}\right)$ and the mean length of the interval.

$$
\operatorname{variance}(\alpha)=\frac{\sigma\left(\left\{d_{i} \mid 1 \leq i<k, d_{i}=p_{i+1}-p_{i}\right\}\right)}{\left(p_{k}-p_{1}\right) /(k-1)}
$$

Definition 4.2 (Density) Density of a repeat $\alpha$ is the proportion of the repeats in the interval between the first and the last occurrences of the repeat. That is,

$$
\operatorname{density}(\alpha)=\frac{(k-1) *|\alpha|}{p_{k}-p_{1}}
$$

where $|\alpha|$ is the number of tokens in $\alpha$.

Generally speaking, machine-generated Web pages often render relevant information in templates that has small variance and large density. To sift for potential patterns, we can use a threshold for each of these measures. A pattern must have a variance less than the variance threshold and a density greater than the density threshold to be considered a candidate pattern.

The above algorithm may fail to extract some layout templates if the variance threshold is not set properly. The reason is that the actual layout templates may have a large variance coefficient. For example, in the output pages of the search engine "Lycos," advertisement banners are inserted among the search results and divide them into several partitions. Such exceptions result in maximal repeats with a large variance. To handle patterns with a variance greater than the specified threshold, the occurrences of a pattern are carefully clustered to see if any partition of the pattern's occurrences can form an independent and regular block. For more details, see [7].

\subsubsection{Composing Extraction Patterns}

As PAT trees compute only repeat substrings, templates with exceptions like missing attributes can not be discovered through PAT trees. We apply the algorithms for multiple string alignment to allow inexact or approximate matching. 
Suppose a candidate pattern has $k$ occurrences, $p_{1}, p_{2}, \ldots, p_{k}$ in the encoded token string. Let string $P_{i}$ denote the string starting at $p_{i}$ and ending at $p_{i+1}-1$. The problem is to find the multiple alignment of the $k-1$ strings $\mathcal{S}=\left\{P_{1}, P_{2}, \ldots, P_{k-1}\right\}$ so that the generalized pattern can be used to extract all records we need. For example, suppose "adc" is the discovered pattern for token string "adcbdadcxbadcxbdadc". Suppose we have the following multiple alignment for strings " $a d c b d$ ", "adcxb" and "adcxbd":

$$
\begin{array}{llllll}
a & d & c & - & b & d \\
a & d & c & x & b & - \\
a & d & c & x & b & d
\end{array}
$$

The extraction pattern can be generalized to cover these three token strings by " $a d c[x \mid-] b[d \mid-]$," where "[ | ]" denotes alternatives and "-" a missing token. This generalization of the patterns allow the data extractor to handle missing attributes that might occur in Web pages [18]. For example, the following permutations of four attributes: $(U, N, A, M),(U, N, A),(U, N, M),(N, A)$ described in [18] are covered by the following generalized pattern " $[U \mid-] N[A \mid-][M \mid-] . "$

Multiple string alignment is a generalization of the alignment problem for two strings that can be solved in $O(n * m)$ by dynamic programming to obtain an optimal edit distance, where $n$ and $m$ are string lengths. Extending dynamic programming for multiple string alignment yields an $O\left(n^{k}\right)$ algorithm. Alternatively, an approximation algorithm is available such that the score of the multiple alignment is no greater than twice the score of the optimal multiple alignment [15]. The approximation algorithm starts by computing the center string $S_{c}$ in $k$ strings that minimizes consensus error. Once the center string is found, each string is then iteratively aligned to the center string to construct multiple alignment, which is in turn used to construct the extraction pattern.

For candidate patterns with density less than one, the center star approximation algorithm [15] for multiple string alignment will be applied to generalize the extraction pattern. Note that the success of this technique lies in the assumption that extraction patterns often occur contiguously. A generalized pattern with $f$ alternatives can match $2^{f}$ permutations of token strings. If the generalization results in extraction patterns with too many alternatives, they will be discarded because an extraction pattern that matches too many token strings is unlikely to be interesting. To restrict the generalization, we set an upper bound of maximum mismatches for the generalized extraction patterns. This upper bound is set to eight in our experiments.

In summary, we can efficiently discover all maximal repeats (with pattern length and occurrence count greater than default thresholds) in the encoded token string by traversing the PAT tree. With variance and density, we can sift the maximal repeats for promising patterns. For patterns with large variance, we can cluster repeats into partitions. As for low density patterns, multiple string alignment is applied to generalize the extraction patterns. The result is a set of candidate patterns of the data records in a Web page. 


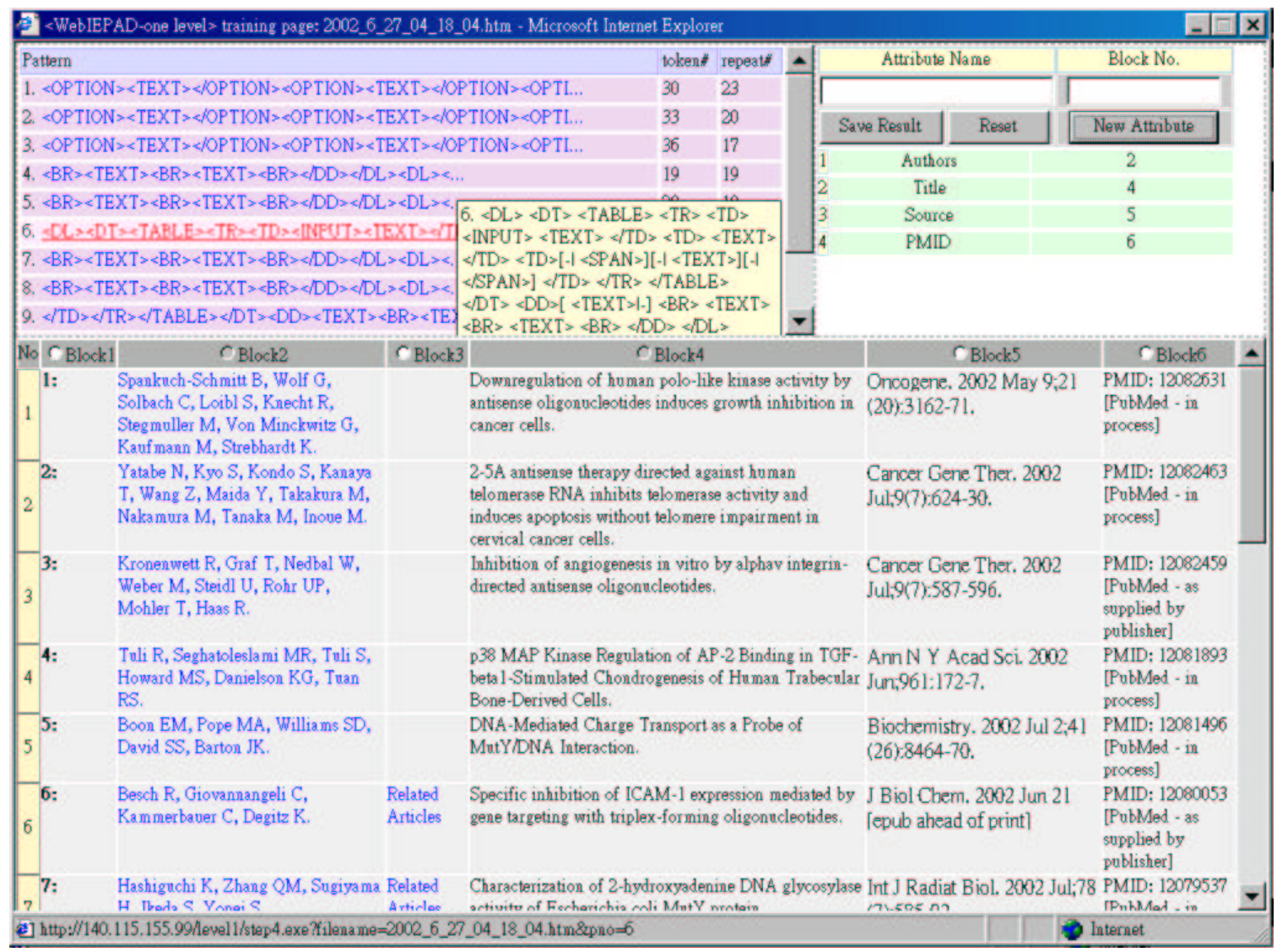

Figure 10: Snapshot of IEPAD

\subsection{Data Alignment for Attribute Extraction}

The pattern discovery algorithm described in the previous subsection allows a data extractor to extract data records from a Web page, whereas it is not sufficient to extract the attributes in a data record. This subsection presents an algorithm for this problem.

Consider a pattern with $m<$ TEXT $>$ tokens. Each $<$ TEXT $>$ token represents a text string that potentially corresponds to an attribute in a data record. For example, since the sixth pattern in the upper-left frame of Figure 10 contains six <TEXT> tokens, each token string that matches this pattern (seven of them are shown in the figure) will be segmented into six blocks (i.e., attributes) as shown in the bottom frame of Figure 10.

The algorithm to segment the set of all records that match a pattern $\alpha$ is outlined in Figure 11. The input to procedure Block_division contains an extraction pattern $\alpha$ and the set of records matched by the pattern. For each record, $r_{i}$, we align the token representation of the record to $\alpha$ (Step 3). As the record might contain missing attributes, this step can extend the token representation of the record to obtain a correct alignment $r_{i}^{\prime}$. Then, for each <TEXT> token in the pattern, extract the contents denoted by the corresponding token in $r_{i}^{\prime}$ (Step 6 to 13). If the corresponding token in $r_{i}^{\prime}$ is a missing token "-", NULL string is used instead. 
Procedure Block_division

Given an extraction pattern $\alpha$

and a set of records $R=\left\{r_{1}, \ldots, r_{n}\right\}$ matched by $\alpha$

1. Begin

2. $\quad$ For each record $r_{i}$ in $\mathrm{R}$ do

3. $\quad r_{i}^{\prime}=\operatorname{Align}\left(\alpha, r_{i}\right) ; / /$ align $r_{i}$ to $\alpha$;

4. $\quad m=0 ; / /$ number of columns;

5. $\quad$ For $j=1$ to $|\alpha|$ do

6. If $\alpha[\mathrm{j}]=<\mathrm{TEXT}>$ or $<\mathrm{A}>$ or $<\mathrm{IMG}>$ then

7. If $r_{i}^{\prime}[j]={ }^{\prime}-^{\prime}$ then

$8 . \quad$ blocks $[\mathrm{i}][\mathrm{m}]=$ null;

$9 . \quad$ else

10. $\quad$ blocks $[\mathrm{i}][\mathrm{m}]=r_{i}^{\prime}[j]$.text;

11. Endif

12. $\quad m=m+1$;

13. Endif

14. Endfor

15. Endfor

16. Return blocks;

17. End

Figure 11: Block division procedure

The above algorithm allows us to segment a data record into attributes if they can be separated with the given encoding scheme. However, sometimes attributes require "finer" segmentation to be extracted correctly. In those cases, we can specify a variety of delimiters as tokens in the encoding scheme and encode the contents of a block repeatedly until an extraction pattern reveals a correct segmentation. For example, the fifth block in the bottom frame of Figure 10 can be further segmented using periods and semicolons as delimiters and then "Oncogene. 2002 May 9; 21(20):3162-71" in the first matched substring can be segmented into three text blocks "Oncogene", "2002 May 9", and "21(20):3162-71."

This can be accomplished by the algorithm Multi-level alignment given in Figure 12. The contents in each block of the input matrix $B M$ are translated through an encoding scheme with finer granularity. Specifically, for each block (column), the procedure encodes the content string in each record (row) by encoding scheme $\beta$ (Step 3 to 6 ). The center-star multiple string alignment algorithm is then applied to compose a consensus pattern (Step 7). Finally, procedure Block_division is invoked to divide the strings into finer subblocks $\left(A_{j}\right)$ according to the consensus pattern (Step 8). Concatenating all subblocks yields a block matrix larger than the input one. The same steps can be repeated until the desired information is successfully separated from the rest of the string. As shown in [7], a two-level encoding is sufficient to extract the target information for most Web data sources in our experiments. The interface of IEPAD will present the aligned data records by columns so that users can select an alignment that correctly segments the data records (see the upper-right frame of Figure 10).

To sum up, generating a data extractor with IEPAD involves the following steps: select an appropriate 
Procedure MultiLevel_alignment

Given a $n \times m$ block $B M$ and an encoding scheme $\pi$

1. Begin

2. For $j=1$ to $m$ do $/ /$ For each column

3. $\quad R=\{\}$;

4. $\quad$ For $i=1$ to $n$ do

5. $\quad r_{i}=$ Encoding $(B M[i][j], \pi) ; / /$ Encode each record

6. $\quad R=R \cup\left\{r_{i}\right\}$;

7. endfor

8. $\quad \beta_{j}=$ CenterStarAlignment $(R) ; / /$ Find the generalized grammar

9. $\quad A_{j}=$ Block_division $\left(\beta_{j}, R\right)$;

10. endfor

11. $A=A_{1}+A_{2}+\ldots+A_{m}$;

12. Return $A$;

13. End

Figure 12: Multi-level alignment

pattern to extract data records (upper-left frame), then select an appropriate alignment to segment data records into attributes (bottom frame) and assign their attribute names (upper-right frame) according to the schema. IEPAD will generate the extraction rules according to the selected pattern and alignment for a data extractor ready to be invoked by the kernel of the WNDL executor.

\section{Related Work}

The vast amount of biological data is available in electronic format with Internet access. However, a fundamental barrier exists because the format of the data and functionality of access routines can vary dramatically from source to source. In this section, we review some previous work in integrated biological databases and compare their capabilities of utilizing online biological databases.

In 1985, Morowitz et al. [28] envisioned the creation of a Biomatrix, in which data, information and knowledge are combined to provide an integrated view of molecular biology. Although Biomatrix has never been completed, the Web of online biological databases has partially fulfilled the promise of Biomatrix. A rough categorization of the approaches that can be applied to fill in its missing parts is given as follows.

- Data warehousing, in which data from various data sources are converted, merged, and stored in a centralized DBMS, e.g., Integrated Genomic Database (IGD) [32] and SRS [5];

- Middleware approaches, in which data are combined from multiple sources without creating a physical warehouse, e.g., BioKleisli [10], TAMBIS [1], and DiscoveryLink [16], etc.

Due to the popularity of the Web, many online biological databases provide services through the Web and establish hyperlinks between related information in different data sources. Migrating all relevant 
data to a data warehouse allows for great flexibility in retrieving and comparing data, but at the cost of losing the established interlinking of the data and the specialized services provided by the original sources. The middleware approaches can potentially exploit the specialized services of a data source so that no functionality is lost when accessing the online databases. However, that depends on the capabilities of their wrappers. Criteria of effective data integration include: first, the capability of transforming the information into a common format; second, the capability of exporting the data for other applications efficiently; third, whether the wrappers can be easily constructed for new data sources and easily modified for changes to the wrapped data sources.

We surveyed three of the most widely used biological data integration systems: BioKleisli, SRS and DiscoveryLink based on the three criteria. The drivers in BioKleisli [10] system are equivalent to the wrappers. BioKleisli offers collection programming language (CPL) to construct a driver. CPL allows for the expression of complex transformation across heterogeneous databases. Sequence Retrieval System (SRS) [5] is an indexed flat file system built on the model of a document retrieval system. SRS converts biological data stored in textual flat files into information in the formats ready for further analysis. Extracted data can be exported by CORBA loaders for client applications. SRS provides a scripting language ICARUS to specify the structure of the data and the syntax for parser generation.

DiscoveryLink [16] consists of a federated database engine and a set of wrappers which translate between query fragments and data source API. DiscoveryLink requires the connection to database servers through ODBC, JDBC, OLE, or native DB2 drivers, etc. The approach of DiscoveryLink satisfies the first two criteria by incorporating specialized wrappers for several database types. Exporting data is not a problem for DiscoveryLink since its wrappers will translate the information into relational data, ready for application buildup.

These previous work, however, offers no standard procedure to directly exploit the specialized services provided by the Web interface of online biological databases. In contrast, our approach is designed to leverage these services. Since the extracted data is transformed into structured, machine-readable formats, the problem of exporting data for other applications becomes insignificant. Nonetheless, our approach is complementary to their work because the Web wrapper agents can be integrated with SRS, BioKleisli, DiscoveryLink and other biological information integration systems to serve as the wrappers to access online databases for their systems.

\section{Conclusion and Future Work}

In this paper, we presented a tool to exploit online biological databases using reconfigurable Web wrapper agents. We described how these agents can be generated and executed based on the script language WNDL and extraction rule generator IEPAD. We demonstrated the utility of this approach with three example applications. We believe this tool can boost the productivity of life scientists and help facilitate biological knowledge discovery. 


\section{Acknowledgements}

We acknowledge the contribution of the members and alumni of AIIA Lab at the Institute of Information Science, Academia Sinica: Hung-Hsuan Huang, Siek Harianto and Elan Hung for implementing and maintaining the early version of the system. This research is supported in part by the National Science Council under grant NSC-90-2213-E-008-053, Taiwan.

\section{Appendix}

Complete WNDL script for PubMed.

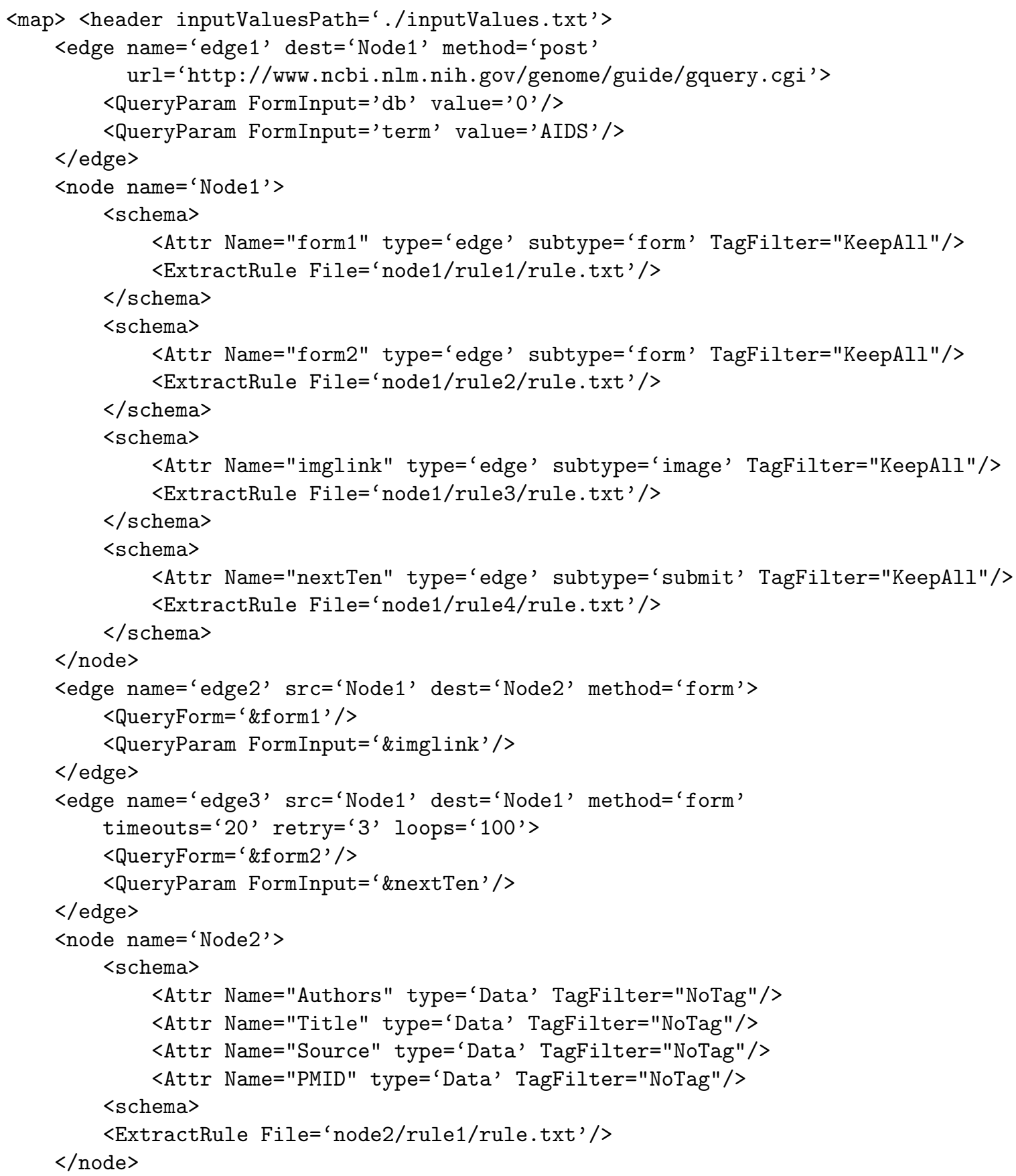


$</ \operatorname{map}>$

\section{References}

[1] Patricia G. Baker, Andy Brass, Sean Bechhofer, Carole Goble, Norman Paton, and Robert Stevens. TAMBIS: Transparent access to multiple bioinformatics information sources. An overview. In Proceedings of the Sixth International Conference on Intelligent Systems for Molecular Biology (ISMB-98), Montreal, pages 25-34, Menlo Park, CA, USA, 1998. AAAI Press.

[2] PDB: Protein Data Bank. http://www.rcsb.org.

[3] NCBI BLAST: Basic Local Alignment Search Tool. http://www.ncbi.nlm.nih.gov/BLAST.

[4] A.D. Baxevanis. The molecular biology database collection: An online compilation of relevant database resources. Nucleic Acids Research, 28(1):1-7, Jan 2000.

[5] P. Carter, T. Coupaye, D. P. Kreil, and T. Etzold. SRS: analyzing and using data from heterogeneous textual databanks. In S. Letovsky, editor, Bioinformatics: Databases and Systems, pages 213-231. Kluwer, Norwell, MA, USA, 1999.

[6] Chia-Hui Chang and Chun-Nan Hsu. Automatic extraction of information blocks using pat trees. In Proceedings of National Computer Symposium, Taipei, Taiwan, 1999.

[7] Chia-Hui Chang, S.-C. Lui, and Y.-C. Wu. Applying pattern mining to web information extraction. In Proceedings of the 5th Pacific-Asia Conference on Knowledge Discovery and Data Mining, pages 4-15, Hong-Kong, 2001.

[8] Chime. http://www.mdli.com/.

[9] World Wide Web Consortium. Web characterization terminology and definitions sheet, May 1999. Working Draft.

[10] Susan B. Davidson, G. Christian Overton, Val Tannen, and Limsoon Wong. Biokleisli: A digital library for biomedical researchers. International Journal on Digital Libraries, 1(1):36-53, 1997.

[11] Robert B. Doorenbos, Oren Etzioni, and Dan S. Weld. A scalable comparison-shopping agent for the world-wide web. In Proceedings of the 1st International Conference on Autonomous Agents, pages 39-48, NewYork, USA, 1997.

[12] Barbara A. Eckman, Jeffrey S. Aaronson, Joseph A. Borkowski, Wendy J. Bailey, Keith O. Elliston, Alan R. Willamson, and Richard A. Blevins. The Merck gene index browser: an extensible data integration system for gene finding, gene characterization and est data mining. Bioinformatics, 14(1):2-13, 1998.

[13] W. Fujibuchi, S. Goto, H. Migimatsu, A. Ogiwara, Y. Akiyama, and M. Kanehisa. DBGET/LinkDB: an integrated database retrieval system. In Pacific Symposium on Biocomputation, volume 3, pages 683-694, 1997.

[14] NCBI GeneBank database and browser. http://www.ncbi.nlm.nih.gov/.

[15] D. Gusfield. Algorithms on Strings, Trees, and Sequences. Cambridge, 1997.

[16] Laura. M. Haas, Peter M. Schwarz, Prasad Kodali, Elon Kotlar, Julia E. Rice, and William C. Swope. DiscoveryLink: A system for integrated access to life sciences data sources. IBM Systems Journal, 40(2):489-511, 2001. Special issue on Deep Computing for the Life Sciences.

[17] Chun-Nan Hsu and Chien-Chi Chang. Finite-state transducers for semi-structured text mining. In Proceedings of IJCAI-99 Workshop on Text mining: Foundations, Techniques and Applications, pages 38-49, Stockholm, Sweden, 1999.

[18] Chun-Nan Hsu and Ming-Tsong Dung. Generating finite-state transducers for semi-structured data extraction from the web. Information Systems, 23(8):521-538, 1998.

[19] Hung-Hsuan Huang. Design and implementation of a configurable wrapper for web information integration. Master's thesis, Department of Computer Science and Information Engineering, National Taiwan University, Taipei, Taiwan, July 2000.

[20] Incyte Genomics Inc. http://reagents.incyte.com/cgi-bin/genome/unigem/unigem.cgi. 
[21] Craig A. Knoblock, Steve Minton, Jose-Luis Ambite, Naveen Ashish, Pragnesh Jay Modi, Ion Muslea, Andrew G. Philpot, and Sheila Tejada. Modeling web sources for information integration. In Proceedings of the 15th National Conference on Artificial Intelligence and Tenth Innovative Applications of Artificial Intelligence Conference (AAAI-98), pages 211-218, Wisconsin, USA, 1998.

[22] Nickolas Kushmerick, Dan Weld, and Robert Doorenbos. Wrapper induction for information extraction. In Proceedings of the 15th International Joint Conference on Artificial Intelligence (IJCAI), pages 729-737, Japan, 1997.

[23] S. Letovsky, editor. Bioinformatics: Databases and Systems. Kluwer, Norwell, MA, USA, 1999.

[24] D.R. Morrison. Patricia-practical algorithm to retrieve information coded in alphanumeric. Journal of $A C M, 15(4): 514-534$, Jan 1968.

[25] Ion Muslea, Steve Minton, and Craig A. Knoblock. A hierarchical approach to wrapper induction. In Proceedings of the 3rd International Conference on Autonomous Agents, pages 190-197, Seattle, WA, 1999.

[26] KEGG: Kyoto Encyclopedia of Genes and Genomes. http://www.genome.ad.jp/kegg/.

[27] SCOP: Structural Classification of Proteins. http://scop.mrc-lmb.cam.ac.uk/scop/.

[28] Committee on models for Biomedical Research. Models for biomedical research: A new perspective. Technical report, National Academy of Sciences, USA, Washington, DC, USA, 1985.

[29] NCBI PubMed database and browser. http://www.ncbi.nlm.nih.gov/PubMed/.

[30] RasMol. http://www.umass.edu/microbio/rasmol/.

[31] PIR: Protein Information Resource. http://pir.georgetown.edu.

[32] O. Ritter, P. Kocab, M. Senger, D Wold, and S. Suhai. Prototype implementation of the integrated genomic database. Computers and Biomedical Research, 27:97-115, 1994.

[33] SWISS-PROT. http://reagents.incyte.com/cgi-bin/genome/unigem/unigem.cgi.

[34] Jeffrey D. Ullman. Principles of Database and Knowledge-base Systems, volume I,II. Computer Science Press, Palo Alto, CA, 1988.

[35] The World-Wide Web Consortium (W3C). Extensible markup language (XML). http://www.w3.org/XML/, 1997.

[36] Gio Wiederhold. Mediators in the architecture of future information systems. IEEE Computer, March 1992. 